\title{
Quantification of Ostwald Ripening in Emulsions via Coarse-Grained
}

\section{Simulations}

\author{
Abeer Khedr and Alberto Striolo* \\ Chemical Engineering Department, University College London, United Kingdom
}

\begin{abstract}
Ostwald ripening is a diffusional mass transfer process that occurs in polydisperse emulsions, often with the result of threatening the emulsion stability. In this work, we design a simulation protocol that is capable of quantifying the process of Ostwald ripening at the molecular level. To achieve experimentally relevant time scales, the Dissipative Particle Dynamics (DPD) simulation protocol is implemented. The simulation parameters are tuned to represent two benzene droplets dispersed in water. The coalescence between the two droplets is prevented via the introduction of membranes, which allow diffusion of benzene from one droplet to the other. The simulation results are quantified in terms of the changes of the droplets volume as a function of time. The results are in qualitative agreement with experiments. The agreement with the Lifshitz-Slyozov-Wagner theory becomes quantitative when the simulated solubility and diffusion coefficient of benzene in water are considered. The effect of two different surfactants was also investigated. In agreement with both experimental observations and theory, the addition of surfactants at moderate concentrations decreased the Ostwald ripening rate because of the reduction in the interfacial tension between benzene and water; as the surfactant film becomes dense, other phenomena are likely to further delay Ostwald ripening. In fact, the results suggest that the surfactant that yields higher density at the benzene-water interface delayed more effectively Ostwald ripening. The formation of micelles can also affect the ripening rate, in qualitative agreement with experiments, although our simulations are not conclusive on such effects. Our simulations show that the coarse-grained DPD formalism is able to capture the molecular phenomena related to Ostwald ripening, and reveal molecularlevel features that could help to understand experimental observations. The results could be useful for predicting, and eventually controlling the long-term stability of emulsions.
\end{abstract}

Keywords: Dissipative particle dynamics, emulsion stability, micelles.

* Author to whom correspondence should be addressed: a.striolo@ucl.ac.uk 


\section{INTRODUCTION}

An emulsion is a system of at least 2 immiscible fluids, yielding one phase in the form of droplets dispersed in a continuous phase. Depending on the droplet size, emulsions are categorized into macro- or micro-emulsions. ${ }^{1}$ In general, emulsions are kinetically stable, but thermodynamically un-stable, as the system free energy is minimised when the two fluids separate and yield two continuous phases. The phase-separation process can be slow, for example because of the presence of surfactants known as emulsifiers. Various surface-active compounds are used for such applications, including solid particles. ${ }^{1-7}$ A kinetically stable emulsion is obtained when the number of droplets, the distribution of their sizes and their arrangement within the continuous phase remain relatively constant within a certain timescale. ${ }^{1}$ Over time, an emulsion is expected to become unstable, and the liquids to phase separate. This can occur by four processes: creaming, flocculation, coalescence and Ostwald ripening. 1, 6 Previous computational studies focused on the effect of surfactants and co-polymers on flocculation and coalescence of emulsion droplets. ${ }^{2,}$, 5, 8, 9 Only few molecular dynamics simulations explicitly considered Ostwald ripening or oil solubilisation into micelles, ${ }^{10-14}$ despite the fact that ripening could be the principal cause for emulsion destabilization in applications such as amorphous drug nanosuspensions and foodstuff. ${ }^{15-20}$ Molecular level observations are required to better understand Ostwald ripening as it occurs in oil-in-water emulsions.

Ostwald ripening occurs due to the solubility difference of dispersed fluids due to different droplet sizes. The Kelvin equation describes how solubility depends on droplet size: ${ }^{21}$

$$
c(r)=c(\infty) \exp \left(\frac{2 \gamma V_{m}}{r R T}\right)
$$

Assuming an oil-in-water emulsion, in Eq. (1), c(r) is the aqueous solubility of the oil (dispersed phase) contained in a droplet of radius $r$, while $\mathrm{c}(\infty)$ is the correspondent solubility at a planar interface. $\gamma$ is the interfacial tension between the two liquids, $V_{m}$ is the molar volume of the dispersed phase, $\mathrm{R}$ is the gas constant and $\mathrm{T}$ the absolute temperature. Because, based on Eq. (1), solubility increases as the droplet radius decreases, the fluid contained in the smaller droplets dissolves preferentially into the continuous phase, where it diffuses to the larger droplets. When the fluid condenses on the surface of the larger droplets, the overall emulsion droplet size increases, eventually yielding a decrease in the total interfacial area. 
Among theories developed to describe Ostwald ripening, the Lifshitz-Slyozov-Wagner (LSW) theory ${ }^{22,23}$ reproduces experimental Ostwald ripening rate data $\left(\omega_{\mathrm{T}}\right)$ for many emulsions, including benzene-in-water ones. ${ }^{24}$ The LSW theory is expressed as:

$$
\omega_{\mathrm{T}}=\frac{\mathrm{dr}_{\text {critical }}{ }^{3}}{\mathrm{dt}}=\frac{8 \gamma \mathrm{C}_{\mathrm{w}}^{\mathrm{eq}} \mathrm{D}_{\mathrm{w}} \mathrm{V}_{\mathrm{m}}}{9 \mathrm{R} \mathrm{T}}
$$

In Eq. (2), $\omega_{\mathrm{T}}$ is estimated by the linear increase of the cube of the 'critical radius' $\left(\mathrm{r}_{\text {critical }}{ }^{3}\right)$ over time. The critical radius $\left(\mathrm{r}_{\text {critical }}\right)$ is defined as the radius of a droplet whose size does not change, while the droplets of radius $\left(r<r_{\text {critical }}\right)$ decrease in size, and droplets of radius $\left(r>r_{\text {critical }}\right)$ increase in size. In Eq. (2), $C_{w}^{e q}$ and $D_{w}$ are the solubility (volume fraction) and the diffusivity of the dispersed phase in the continuous one, expressed in $\mathrm{m}^{3} / \mathrm{m}^{3}$ and $\mathrm{cm}^{2} / \mathrm{s}$, respectively. Finally, in Eq. (2) $\gamma$ is the interfacial tension between the two liquids. Eq. (2) provides an expression of the Ostwald ripening rate for dilute emulsions, where the effect of the dispersed phase concentration is not considered. In fact, the Ostwald ripening rate can increase when the dispersed phase volume fraction increased from 0 to $0.3 .^{25} \mathrm{Kabalnov}$ et al., e.g., ${ }^{26}$ included a correction factor of 1.75 when the oil volume fraction increased to 0.1 .

Kabalnov et al. ${ }^{24}$ showed that the LSW theory satisfactorily reproduces experimental Ostwald ripening rates for several oil-in-water emulsions, including as oil 1,2-dichloreoethane, benzene, nitrobenzene, toluene, and p-xylene. They found that the critical radius coincides with the mean droplet radius $(\bar{r})$, and that this value changes over time. This group showed that increasing the concentration of the surfactant sodium dodecyl sulfate (SDS) within 1,2-dichloroethane-inwater emulsions reduces the interfacial tension from $31 \mathrm{mN} / \mathrm{m}$ (when no surfactant is present) to $3.8 \mathrm{mN} / \mathrm{m}$ at $0.1 \mathrm{M}$ surfactant concentration, which would decrease the Ostwald ripening rate by $\sim$ one order of magnitude. In a further study, Kabalnov et al. ${ }^{26}$ studied the dependency of the Ostwald ripening rate on the aqueous solubility in water of a series of $\mathrm{C}_{9}-\mathrm{C}_{16}$ aliphatic hydrocarbons stabilized by SDS. The Ostwald ripening rate decreases by $\sim$ one order of magnitude when the oil chain length increased by two $\mathrm{CH}_{2}$ groups, because of the reduced solubility in water. Although they also observed oil solubilisation into the core of surfactant micelles, they concluded that such phenomenon has negligible effect on Ostwald ripening rate. In a further investigation, ${ }^{27}$ no changes in Ostwald ripening rate were reported as SDS concentration was increased from 0.03 to $1 \mathrm{M}$ (the SDS CMC is $\sim 0.0082 \mathrm{M}$ ). Taylor et al. ${ }^{28}$ reported that the Ostwald ripening rate falls linearly as the SDS concentration increases to the $\mathrm{CMC}$, due to the decrease in the interfacial tension, and then it increases very slightly above it. 
It was suggested that the micelles have a negligible effect on the ripening rate because of their short lifetime: when the micelles break, they release the oil in the aqueous phase.

In a series of reports, McClements and his group ${ }^{19,20,29-33}$ described the dependency of the Ostwald ripening rate on surfactant concentration. With the aid of static light scattering, ${ }^{29}$ they reported that micelles of the non-ionic surfactant Tween 20 formed micelles with oil in their core. The results showed an increase in the droplet size, contrary to expectations. The results were explained as follows: oil solubilisation in micelles caused some droplets to become smaller; these smaller droplets favoured Ostwald ripening, leading to the formation of the larger droplets observed. These authors reported that short unsaturated hydrocarbons have higher solubilisation rate and maximum solubility capacity in the micelles compared to long saturated ones. McClements et al. ${ }^{33}$ investigated the Oswald ripening rate as a function of surfactant concentration for SDS (ionic) and Tween 20, Brij 35 and Triton X-100 (non-ionic) above their CMC. In these experiments the oil was n-tetradecane, at $5 \mathrm{wt}$. \%. They found a clear dependency of the Ostwald ripening rate on the interfacial tension for non-ionic surfactants. However, the Ostwald ripening rate was the slowest in the presence of SDS, although this surfactant did not yield the lowest interfacial tension. They suggested that surfactants affect the Ostwald ripening rate via their effect on the interfacial tension, and also via the effective interactions between micelles and the droplet surface. For example, in the case of ionic surfactants, the electrostatic repulsion between micelles and droplets surface could prevent oil exchange between the two. Finally, they suggested that the maximum solubilizing capacity of micelles could affect Ostwald ripening, although the results were not conclusive. They found no clear dependency of the Ostwald ripening rate on the solubilisation kinetics or on the solubilisation capacity of micelles of non-ionic surfactants for emulsions containing ntetradecane. ${ }^{30,31}$

Ariyaprakai et al. ${ }^{34}$ reported that the presence of micelles enhanced the rate of Ostwald ripening for different surfactants by factors of up to 50, depending on the surfactant type and concentration. They studied Ostwald ripening of decane-in-water emulsions stabilized by dodecyl ether surfactants with different ethylene oxide (EO) chain lengths: $\mathrm{C}_{12} \mathrm{E}_{7}, \mathrm{C}_{12} \mathrm{E}_{8}, \mathrm{C}_{12} \mathrm{E}_{10}$ and Brij 35 (with 23 EO groups). The results showed that the repulsion between micelles and droplet surfaces, due to different hydrophilic groups, has no systematic effect on the Ostwald ripening rate. In fact, Ariyaprakai et al. suggested that micelle collision and coalescence with the droplets does not contribute significantly to Ostwald ripening, while the observed increase 
in Ostwald ripening rate is due to the enhanced oil transport across the aqueous phase facilitated by the ability of micelles to become supersaturated with oil.

Han et al. ${ }^{16}$ quantified how Ostwald ripening depends on the characteristic of the interfacial membrane formed by surfactants adsorbed on the droplet surfaces. They quantified the performance of non-ionic polyethylene alkyl ether surfactants as stabilizers for flavour oil emulsions. The surfactants considered were S10 (polyoxyethylene 10 stearyl ether), S20 (polyoxyethylene 20 stearyl ether), L23 (polyoxyethylene 23 lauryl ether) and S100 (polyoxyethylene 100 stearyl ether). Out of these surfactants, S10 was effective at preventing Ostwald ripening, while others, including S100, were not. While no systematic trend was observed, the results were explained by the ability of S10 to yield a dense interfacial membrane, which could delay oil transport. McClements et al. ${ }^{35}$ reported that SDS-chitosan films could delay ripening of n-tetradecane droplets in water, because the thickness of the interfacial film can provide mechanical resistance to droplets shrinkage and growth. These findings show that the density and the thickness of the interfacial membrane could play a major role on Ostwald ripening and on the stability of emulsions, although systematic trends as a function of surfactants molecular architecture might not be evident because different phenomena take place simultaneously.

Several computational studies related to this topic have also been reported. Karaborni et al., ${ }^{10,11}$ for example, studied the solubilisation of oil in aqueous micelles using molecular dynamics (MD) simulations. The oil was an equimolar mixture of two components with different chain lengths. ${ }^{11}$ Increasing the chain length was found to decrease the molecular exchange between droplets and aqueous surfactant phase, due to the decrease of oil solubility in water, which agrees with previous experimental observations..$^{29,34}$ In fact, oil molecules were found to diffuse from the droplet to the aqueous phase, and then to the micelle core. Transport of oil molecules from the droplet to the micelles was also detected upon collision between micelles and the droplet. Karaborni et al. described the 'budding-off' mechanism, according to which adsorbed surfactants depart the droplet surface with some oil molecules, yielding a micelle. ${ }^{10}$ The three mechanisms observed during the simulations correspond to those generally considered to interpret experimental data. ${ }^{29,30,34}$ Mondal el al. ${ }^{12}$ carried out MD simulations to study the solubilisation of aromatic compounds such as benzene, toluene, pyridine and phenol in SDS micelles. They found that pyridine and phenol are solubilized in the Stern layer and the micelle-water interface while non-polar aromatics (benzene and toluene) tend to accumulate in 
the palisade and inner core of the micelles. The type of the aromatics also affected the shape of the micelles, with polar aromatics deforming the SDS micelles.

Directly simulating Ostwald ripening has been attempted only in idealised systems, presumably because of the high computational requirements. For example, Jung et al., ${ }^{36}$ while studying droplet formation and growth in dilute Lennard-Jones (LJ) fluids using Brownian dynamics (BD) simulations, reported evidence of Ostwald ripening. Jung et al. ${ }^{37}$ also studied the growth of LJ fluid droplets dispersed in a polymer network using MD. Ostwald ripening was observed when the polymer network had an intermediate mesh size, because when the mesh size was too small, the droplets could not form, while when the mesh size was large, droplets calescence occurred. Kraska ${ }^{14}$ explicitly simulated Ostwald ripening using MD, but for two argon clusters dispersed in vapour. To retard coalescence between the clusters, Ostwald ripening was accelerated via adding biases to the system. Zhang et al. ${ }^{38}$ simulated coarsening and crystallization of solid particles, and reported that after aggregation, nanoparticles started to coarsen due to atomic diffusion. The two nanoparticles were however at contact. Other computational attempts to understand Ostwald ripening were conducted using phase-field simulations. ${ }^{39,40}$

To complement the experimental observations in the literature, and to provide synergistic molecular-level insights, we introduce here a simulation set up to directly simulate Ostwald ripening for an oil-in-water emulsion. We employ an algorithm specifically designed to prevent creaming, flocculation and coalescence, so that Ostwald ripening is the only mechanism allowed for breaking the emulsion. We select benzene-in-water emulsions because Kabalnov et al. ${ }^{24}$ reported both experimental and theoretical results for a similar system. Once the capability of our algorithm to replicate Ostwald ripening is demonstrated, we quantify how surfactants affect the Ostwald ripening rate. We select surfactant concentrations at which the formation of micelles is not likely. Focus is on the effect of the hydrophilic group length, and on the effect that could be due to the density and the thickness of the interfacial surfactant film. We consider two surfactants with different hydrophilic groups, and compare the results to experimental data reported by Ariyaprakai et al., ${ }^{34}$ Han et al., ${ }^{16}$ and McClements et al. ${ }^{35}$

To overcome computing-power limitations, we implement a coarse-grained computational framework. Instead of describing each molecule with atomic precision, a group of atoms/molecules are considered as a single entity, a 'bead', which reduces computational demands. We select the Dissipative Particle Dynamics methodology (DPD) because it allows 
us to investigate relatively large systems over time scales that are experimentally relevant. DPD has been used previously to investigate emulsions ${ }^{41-45}$ and complex systems. ${ }^{46-49}$ In our previous work, ${ }^{46}$ we derived DPD interaction parameters to reproduce the interfacial tension for the water/benzene interface, as well as to describe the CMC of two non-ionic surfactants representative of the aqueous octyl polyethylene oxide $\mathrm{C}_{8} \mathrm{H}_{17} \mathrm{O}\left(\mathrm{C}_{2} \mathrm{H}_{4} \mathrm{O}\right)_{\mathrm{m}} \mathrm{H}$ family. The parameters used in the present study were taken from our prior work.

The remainder of the manuscript is organized as follows: in Section 2 we briefly summarise the simulation methodology, algorithms and parameters. In Section 3 we summarise our main simulation results and we discuss how they favourably compare to experimental and theoretical observations from literature. We then conclude highlighting the main outcomes of this work, including some limitations of the method proposed.

\section{Simulation Models and Algorithms}

\subsection{Dissipative Particle Dynamics}

DPD is a coarse-grained technique, in which beads represent a few molecules of the different components. The framework was introduced by Hoogerbrugge and Koelman in $1992 .{ }^{50}$ Within DPD, the total force acting on one bead, $i,\left(F_{i}\right)$ is given by the contribution of conservative $\mathbf{f}^{\mathrm{C}}\left(\mathbf{r}_{\mathrm{ij}}\right)$, dissipative $\mathbf{f}^{\mathrm{D}}\left(\mathbf{r}_{\mathrm{ij}}, \mathbf{v}_{\mathrm{ij}}\right)$, random $\mathbf{f}^{\mathrm{R}}\left(\mathbf{r}_{\mathrm{ij}}\right)$, and bonding $\mathbf{f}^{\mathrm{S}}\left(\mathbf{r}_{\mathrm{ij}}\right)$ forces. In DPD, the total force acting on a bead is a summation of the four types of pair interactions: ${ }^{51,52}$

$$
\mathbf{F}_{\mathrm{i}}=\sum_{\mathrm{j} \neq \mathrm{i}}\left[\mathbf{f}^{\mathrm{C}}\left(\mathbf{r}_{\mathrm{ij}}\right)+\mathbf{f}^{\mathrm{D}}\left(\mathbf{r}_{\mathrm{ij}}, \mathbf{v}_{\mathrm{ij}}\right)+\mathbf{f}^{\mathrm{R}}\left(\mathbf{r}_{\mathrm{ij}}\right)+\mathbf{f}^{\mathrm{S}}\left(\mathbf{r}_{\mathrm{ij}}\right)\right]
$$

The conservative force between two beads $\mathrm{i}$ and $\mathrm{j}$ is defined by a repulsion interaction parameter $a_{\mathrm{ij}}$, which depends on the distance between the two beads according to Eq. (4):

$$
\mathbf{f}^{\mathrm{C}}\left(\mathbf{r}_{\mathrm{ij}}\right)= \begin{cases}a_{\mathrm{ij}}\left(1-\mathrm{r}_{\mathrm{ij}} / \mathrm{r}_{\mathrm{c}}\right) \hat{\mathbf{r}}_{\mathrm{ij}} & \mathrm{r}_{\mathrm{ij}}<\mathrm{r}_{\mathrm{C}} \\ 0 & \mathrm{r}_{\mathrm{ij}} \geq \mathrm{r}_{\mathrm{C}}\end{cases}
$$

The dissipative and the random forces describe the Brownian motion of beads in DPD, and are expressed as:

$$
\begin{gathered}
\mathbf{f}^{\mathrm{D}}\left(\mathbf{r}_{i j}, \mathbf{v}_{\mathrm{ij}}\right)=-\gamma \omega^{\mathrm{D}}\left(\mathrm{r}_{\mathrm{ij}}\right)\left(\hat{\mathbf{r}}_{\mathrm{ij}} \cdot \mathbf{v}_{\mathrm{ij}}\right) \hat{\mathbf{r}}_{\mathrm{ij}} \\
\mathbf{f}^{\mathrm{R}}\left(\mathbf{r}_{\mathrm{ij}}\right)=\sigma \omega^{\mathrm{R}}\left(\mathrm{r}_{\mathrm{ij}}\right) \xi_{\mathrm{ij}} \Delta \mathrm{t}^{-1 / 2} \hat{\mathbf{r}}_{\mathrm{ij}}
\end{gathered}
$$


The vector $\mathbf{v}_{\mathrm{ij}}$ is the relative velocity $\left(\mathbf{v}_{\mathrm{ij}}=\mathbf{v}_{\mathrm{i}}-\mathbf{v}_{\mathrm{j}}\right)$ between beads $\mathrm{i}$ and $\mathrm{j}$. The variation of the friction coefficient and random force with distance are described by $\omega^{D}\left(r_{i j}\right)$ and $\omega^{R}\left(r_{i j}\right)$, respectively. $\xi_{\mathrm{ij}}$ is a random number selected following a Gaussian distribution with zero mean and unit variance; $\gamma$ is a coefficient controlling the strength of the frictional forces between the DPD beads; $\sigma$ determines the magnitude of the random pair force between particles. In order to conserve the Gibbs equilibrium conditions, $\omega^{\mathrm{D}}\left(\mathrm{r}_{\mathrm{ij}}\right)$ and $\omega^{\mathrm{R}}\left(\mathrm{r}_{\mathrm{ij}}\right)$, $\sigma$ and $\mathrm{y}$ are related to each other by the following constraints:

$$
\begin{gathered}
\omega^{\mathrm{D}}\left(\mathrm{r}_{\mathrm{ij}}\right)=\left[\omega^{\mathrm{R}}\left(\mathrm{r}_{\mathrm{ij}}\right)\right]^{2} \\
\sigma^{2}=2 \mathrm{k}_{\mathrm{B}} \mathrm{T \gamma}
\end{gathered}
$$

To describe chemical bonds within a molecule, spring forces are introduced, which are described by $\mathrm{k}_{\mathrm{s}}$, the spring constant, and $\mathrm{r}_{0}$, the equilibrium bond length.

$$
\mathbf{f}^{\mathrm{S}}\left(\mathbf{r}_{\mathrm{ij}}\right)=-\mathrm{k}_{\mathrm{s}}\left(\mathrm{r}_{\mathrm{ij}}-\mathrm{r}_{0}\right) \hat{\mathbf{r}}_{\mathrm{ij}}
$$

All the DPD parameters used in this work are taken from our previous study, ${ }^{46}$ where we showed that they are able to reproduce water-benzene interfacial tension, as well as some of the properties of the octyl polyethylene oxide $\mathrm{C}_{8} \mathrm{H}_{17} \mathrm{O}\left(\mathrm{C}_{2} \mathrm{H}_{4} \mathrm{O}\right)_{\mathrm{m}} \mathrm{H}$ aqueous non-ionic surfactants. All the repulsion parameters used are listed in Table 1. The other parameters are discussed in Section 2.2.

\subsection{Computational Details}

All simulations presented here are conducted using the simulation package LAMMPS,${ }^{53}$ with the isothermal pair style DPD force fields.

Simulations were performed in a rectangular box of dimensions $74 \times 36 \times 36 \mathrm{r}_{\mathrm{c}}{ }^{3}\left(\mathrm{~L}_{\mathrm{x}} \times \mathrm{L}_{\mathrm{y}} \times\right.$ $\left.\mathrm{L}_{\mathrm{z}}\right)$, which is shown in Figure 1. The degree of coarse graining $\left(\mathrm{N}_{\mathrm{m}}\right)$ is chosen to be 5 , which means that each DPD water bead represents 5 water molecules. Thus, the volume of one DPD bead $\left(V_{\text {bead }}\right)$ equals $150 \AA^{3}$. The density $\left(\rho_{\text {DPD }}\right)$ in the simulation box is taken as 3 . The cutoff distance $\left(r_{c}\right)$ is taken as 1 , and according to the relation $r_{c}=\sqrt[3]{\rho_{\text {DPD }} V_{\text {bead }}}, r_{c}$ is equal to $7.66 \AA$ and the diameter of one bead equals $0.86 \mathrm{r}_{\mathrm{c}}$. The time step $\Delta \mathrm{t}=0.04 \tau$ is used to integrate the equations of motion. The correspondence between simulation time and real time can be estimated by comparing the experimental diffusion coefficient of water $\mathrm{D}_{\text {water }}(2.43 \times$ $10^{-5} \mathrm{~cm}^{2} / \mathrm{s}$ ) to the correspondent value for the water beads in the DPD simulation, $\mathrm{D}_{\text {sim }}$, as shown in Eq. (10). ${ }^{54}$ Following standard protocols, the simulated diffusion coefficient is 
calculated by the slope of the mean square displacement (MSD) of the water beads against time (in $\mathrm{r}_{\mathrm{c}}{ }^{2} / \tau$ ), as described by Eq. (11). This results in a time step $\Delta \mathrm{t}=5.45 \mathrm{ps}$. For further details, we refer to our prior work. ${ }^{46}$

$$
\begin{gathered}
\tau=\frac{N_{m} D_{\text {sim }} r_{c}{ }^{2}}{D_{\text {water }}} \\
D_{\text {sim }}=\frac{M S D}{6 t}
\end{gathered}
$$

To study Ostwald ripening, it is necessary to prevent flocculation or coalescence between the droplets. Thus, membranes were inserted between the two droplets. Note that two membranes are required because of periodic boundary conditions. Each membrane has channels large enough to allow molecular exchange between the droplets, yet small enough to prevent the droplets from diffusing across the membrane. The height of each channel is equal to $5 \mathrm{r}_{\mathrm{c}}$. The interaction between the membrane and water beads is equal to the self repulsion parameter $\left(a_{\text {membrane-water }}=131.5\right)$ to facilitate the passage of water through the chanels, while the repulsion parameter between the membrane and the other constituent is set to a much higher value ( $a_{\text {membrane-oil }}=200$ and $a_{\text {membrane-surfactant }}=400$ ) to prevent any interaction with the membrane. The parameters are reported in Table 1.

Two benzene droplets are inserted in water. To observe ripening, it is necessary that the droplets have different sizes. The droplets are of diameter 9.7 (droplet 1) and $26.15 \mathrm{r}_{\mathrm{c}}$ (droplet 2), equivalent to 7.4 and $20.04 \mathrm{~nm}$, respectively. The number of benzene beads in the two droplets at the beginning of the simulations are 1385 and 27984, respectively. 


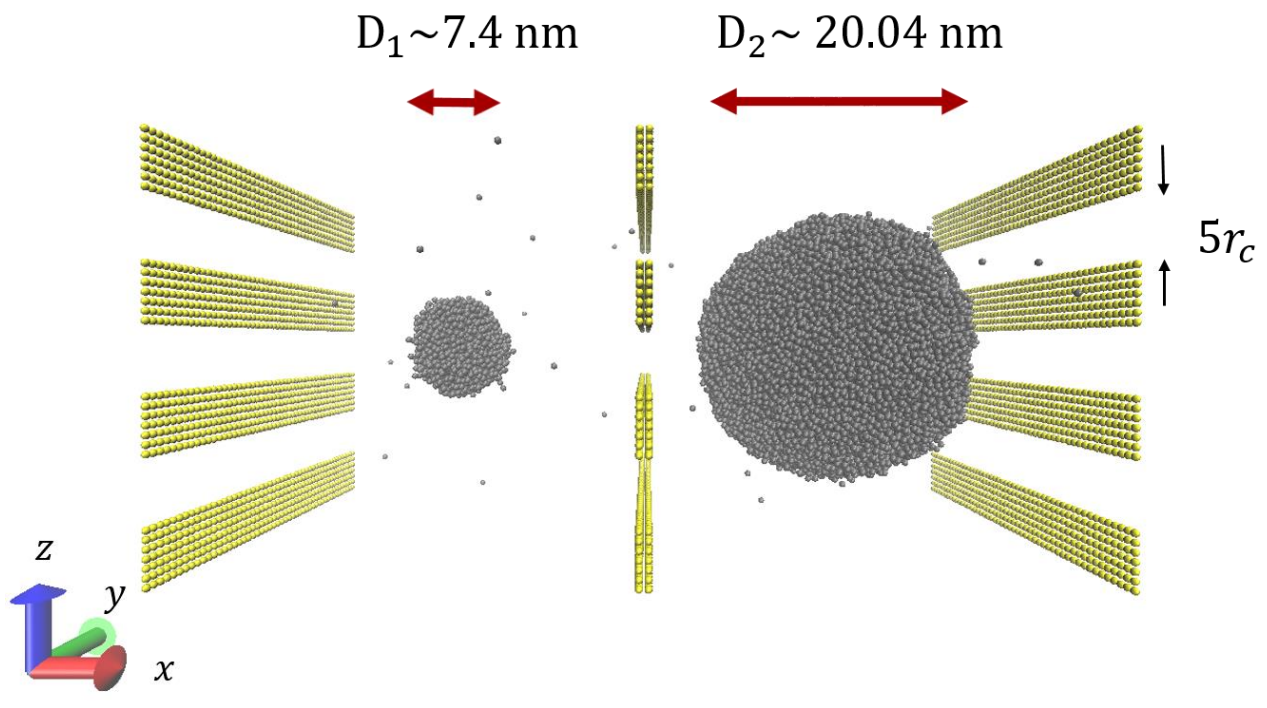

Figure 1. Two oil droplets (grey beads) in water (transparent), separated by membranes (yellow beads) located in the middle and at the edges.

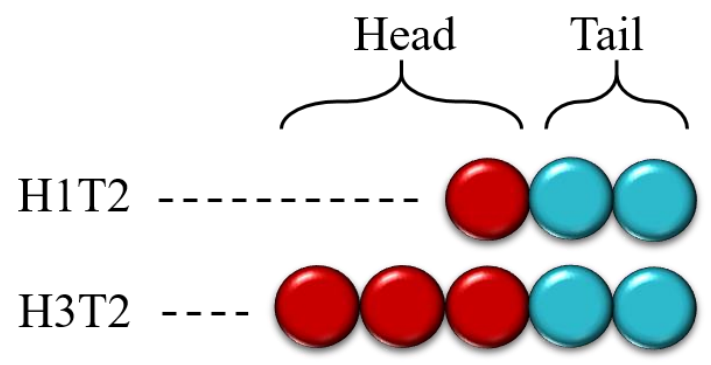

Figure 2. A schematic representation of the surfactant molecules used in this study. The surfactant with one red bead as hydrophilic group (head) is denoted as H1T2 and the other molecule with three beads as a head is denoted as H3T2.

Table 1. DPD repulsion parameters.

$\begin{array}{cccccc} & \text { Water } & \text { Benzene } & \text { Head } & \text { Tail } & \text { Membrane } \\ \text { Water } & 131.5 & 171.43 & 0 & 168.9 & 131.5 \\ \text { Benzene } & & 131.5 & 142.07 & 132.01 & 200 \\ \text { Head } & & 131.5 & 140.83 & 400 \\ \text { Tail } & & & 131.5 & 400 \\ \text { Membrane } & & & & 131.5\end{array}$


To quantify Ostwald ripening, it is required to identify and count the oil beads that belong to a certain droplet / cluster. We follow the approach proposed by Johnston and co-workers, ${ }^{55}$ as implemented in our previous study. ${ }^{46}$ In this approach, two surfactants belong to the same droplet / micelle when the distance between any of their hydrophobic tail beads is lower than $1 \mathrm{r}_{\mathrm{c}}$. If an oil or tail bead is separated by a distance shorter than $1 \mathrm{r}_{\mathrm{c}}$ from any of the oil beads in a droplet, they are considered as part of that droplet.

The radius of the droplet was estimated from the radius of gyration according to:

$$
\mathrm{r}_{\mathrm{GYR}}^{2}=\frac{3}{5} \mathrm{r}^{2}
$$

To estimate the radius of gyration, we require the gyration tensor, which is defined as: ${ }^{56,57}$

$$
\mathcal{T}_{\mathrm{GYR}}=\frac{1}{\mathrm{~N}}\left[\begin{array}{ccc}
\sum \mathrm{x}_{\mathrm{i}}{ }^{2} & \sum \mathrm{x}_{\mathrm{i}} \mathrm{y}_{\mathrm{i}} & \sum \mathrm{x}_{\mathrm{i}} \mathrm{z}_{\mathrm{i}} \\
\sum \mathrm{x}_{\mathrm{i}} \mathrm{y}_{\mathrm{i}} & \sum \mathrm{y}_{\mathrm{i}}{ }^{2} & \sum \mathrm{y}_{\mathrm{i}} \mathrm{z}_{\mathrm{i}} \\
\sum \mathrm{x}_{\mathrm{i}} \mathrm{z}_{\mathrm{i}} & \sum \mathrm{y}_{\mathrm{i}} \mathrm{z}_{\mathrm{i}} & \sum \mathrm{z}_{\mathrm{i}}{ }^{2}
\end{array}\right]
$$

The summations in Eq. (13) are performed over $\mathrm{N}$ droplet beads, whose $\mathrm{x}, \mathrm{y}$ and $\mathrm{z}$ coordinates are related to the droplet centre. By the diagonalization of the gyration tensor, we obtain:

$$
\mathcal{T}_{\text {GYR }}^{\text {diag }}=\left[\begin{array}{ccc}
\mathrm{S}_{1}{ }^{2} & 0 & 0 \\
0 & \mathrm{~S}_{2}{ }^{2} & 0 \\
0 & 0 & \mathrm{~S}_{3}{ }^{2}
\end{array}\right]
$$

The radius of gyration is related to the Eigen values, according to:

$$
\mathrm{r}_{\mathrm{GYR}}{ }^{2}=\mathrm{S}_{1}{ }^{2}+\mathrm{S}_{2}{ }^{2}+\mathrm{S}_{3}{ }^{2}
$$

To calculate the radius of gyration, each droplet was simulated in isolation, surrounded by solvent, within separate simulations. Each of these droplets were simulated for $10^{5}$ steps; during these simulations, one frame was extracted every 1000 step, yielding 100 frames. The radius of gyration reported below was the average over these 100 frames.

Two different surfactants were used, with short and long hydrophilic head-groups, respectively. The surfactants are shown schematically in Figure 2. Consecutive beads in the surfactant molecule are connected with harmonic springs (see Eq. (9)) having an equilibrium bond length $\mathrm{r}_{0}=0.9 \mathrm{r}_{\mathrm{c}}$ and spring constant $\mathrm{k}_{\mathrm{s}}=100 \mathrm{k}_{\mathrm{B}} \mathrm{T} / \mathrm{r}_{\mathrm{c}}{ }^{2}{ }^{58}$ The force field parameters used to describe the beads in the surfactant molecules are reproduced in Table 1. To mimic the experimental procedure of introducing surfactants to emulsions, surfactants were added to water (solvent) at different positions within the simulation box. The initial configurations are 
shown in Figure 3. Starting our simulations from different initial configurations yielded consistent results. The results obtained from initial configurations shown in panels (b) and (e) are shown in this manuscript, while a comparison between all results is included in the Supplementary Information.

To quantify the number of surfactant molecules needed to saturate both droplet surfaces, we introduced a large number of surfactants in the system, and we monitored, as a function of time, the amounts of surfactants adsorbed on each droplet. Once a plateau was reached, the correspondent amount was considered to be the surface coverage at saturation. Results are discussed in Section 3, with additional details provided in Figure S4 to Figure S6 in Supplementary Information.

a)

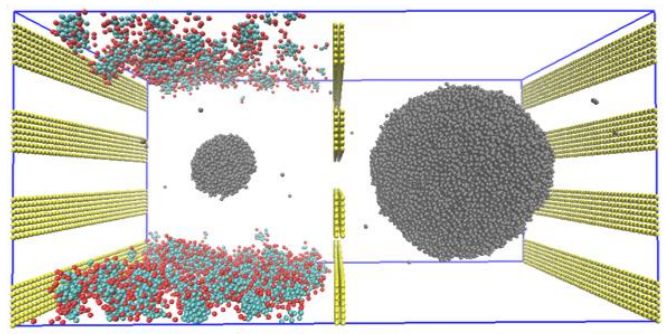

b)

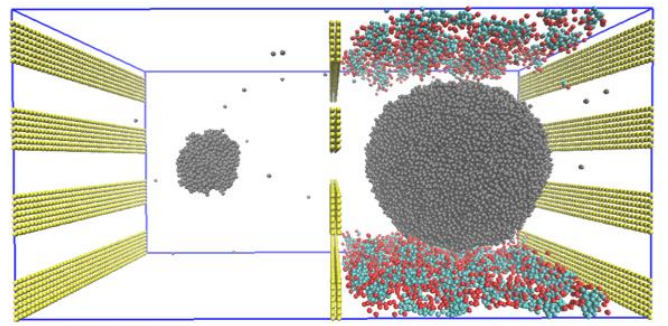

c)

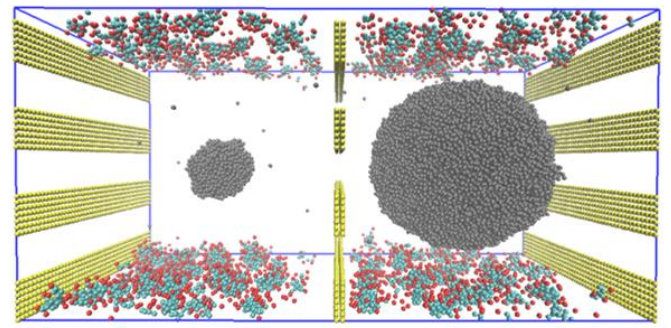

d)

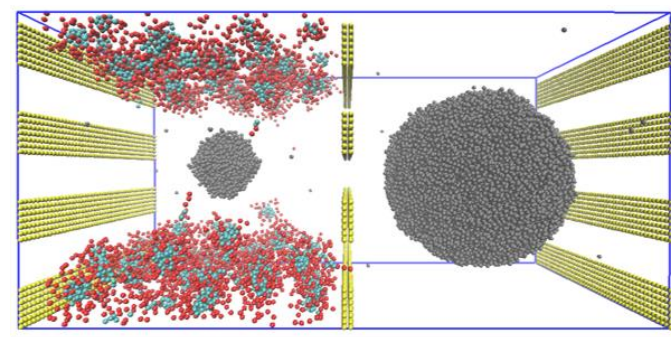

e)

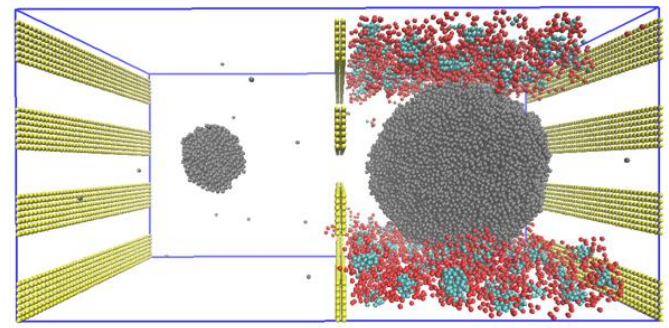

f)

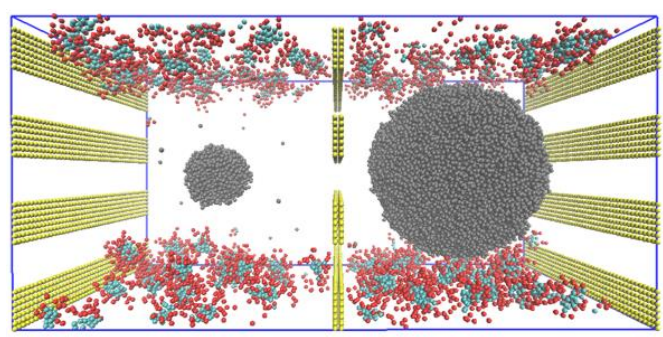

Figure 3. Three initial configurations for the benzene-in-water droplets simulated in the presence of H1T2 (panels a, b and c) and H3T2 (panels d, e and f).

Additional simulations were conducted to extract the parameters used in Eq. (2). Explicitly, the solubility of simulated benzene in water was calculated by estimating the ratio 
of the benzene beads dissolved in bulk water to the number of bulk water beads. The results were averaged over $10^{6}$ simulation steps, which is equivalent to $5.45 \mu \mathrm{sec}$.

To estimate the simulated oil beads diffusivity in water $\left(D_{\text {oil in water }}\right)$, we calculated the diffusion coefficient $\left(\mathrm{D}_{\text {sim }}\right)$ from the mean square displacement (MSD) of oil beads in water (see Eq. (11)). The reported value of $D_{\text {sim }}$ is the average over three simulations, each running for $10^{6}$ steps. Then, knowing that in the simulated system $\tau=136.3 \mathrm{ps}$, ${ }^{46}$ we calculated the diffusion coefficient $\mathrm{D}_{\text {oil in water }}$ as:

$$
\tau=\frac{N_{m} D_{\text {sim }} r_{c}{ }^{2}}{D_{\text {oil in water }}}
$$

To apply the LSW theory to the simulation results it is important to describe how the interfacial tension depends on droplet curvature: ${ }^{59}$

$$
\gamma=\gamma_{0} /\left(1+2 \delta / R_{S}\right)
$$

In Eq. (17), $\gamma$ and $\gamma_{0}$ are the interfacial tensions at curved and planar interface, respectively; $R_{S}$ is the droplet radius; $\delta$ is the Tolman length, $\sim 10^{-8} \mathrm{~cm} .{ }^{59,} 60$ Based on our prior results, ${ }^{46}$ our DPD parameters yield $\gamma_{0}=30 \pm 8 \mathrm{mN} / \mathrm{m}$ for a water-benzene planar interface. Using Eq. (17), we estimate in $28.5 \pm 8$ and $29.4 \pm 8 \mathrm{mN} / \mathrm{m} \gamma$ for droplets 1 and 2, respectively. Due to the small differences between $\gamma$ and $\gamma_{0}$, we used $\gamma_{0}$ for the calculations below.

\section{Simulation Results}

\subsection{Ostwald Ripening for Benzene Droplets Dispersed in Water}

The size of the two simulated benzene droplets, expressed as number of beads per droplet, was monitored during simulations that lasted up to $48 \times 10^{6}$ steps, equivalent to $262 \mu \mathrm{sec}$. The results are shown in Figure 4, in which the two horizontal dashed lines indicate the sizes of the two droplets at the beginning of the simulation, provided for reference. It can be seen that the simulations were long enough for the smaller droplet, droplet 1, to completely disappear, when all its beads diffused to the larger droplet 2. Qualitatively, these results demonstrate that the DPD formalism is able to capture the fundamental phenomena responsible for Ostwald 
ripening. To ensure the reliability of the results, additional simulations were carried out for the same system, but starting from the two benzene droplets with sizes similar to the ones obtain after $94.7 \mu \mathrm{sec}$ of the simulation just discussed. The results, shown in Figure $\mathrm{S} 1$ in Supplementary Information (SI), are consistent with those presented in Figure 4.

Because of experimental limitations faced when attempting to track the molecular exchange between droplets in an emulsion, evidence of Ostwald ripening is obtained experimentally by collecting size distributions of emulsion droplets, and by studying changes in the mean droplet size over time. To compare our simulation results with experiments and theory, we plot in Figure 5 the cube of the mean radius of droplet 2 as a function of time. The droplet radius is estimated following the procedure discussed in Section 2.2. The results (Figure 5) show a linear relation with slope $0.17 \pm 0.01 \mathrm{~nm}^{3} / \mu \mathrm{sec}$, in agreement with Eq. (2).

A comparison of the simulated Ostwald ripening rate vs. experimental data and theoretical predictions is presented in Table 2. The predictions are those by Kabalnov et al., ${ }^{24}$ who employed the LSW theory at $20^{\circ} \mathrm{C}$. To predict the Ostwald ripening rate at $25^{\circ} \mathrm{C}$, we use the LSW theory (Eq. (2)) with the experimental values listed in Table 3. The comparison provided by Table 2 shows that our simulations underestimate the Ostwald ripening rate by one order of magnitude compared to experimental data and theoretical estimations. To reconcile this difference, we apply the LSW theory (Eq. (2)) using solubility and diffusivity of benzene beads in water as estimated from our simulations (see Section 2.2 for a discussion on methods). Note that the simulations underestimate the solubility of benzene in water by two orders of magnitude compared to experiments, while they overestimate benzene diffusivity in water by a factor of 2 (see Table 3). These differences are in part due to the soft potentials implemented in the DPD framework. While such potentials allow us to achieve relatively long simulation times, they yield un-naturally high transport properties. Perhaps other coarse-grained simulation approaches could mitigate such limitations. However, it should be noted that, when these simulation data are used in the LSW theory (Eq. (2)), along with the simulated benzenewater interfacial tension from our prior work $(\sim 30 \mathrm{mN} / \mathrm{m}),{ }^{46}$ we predict an Ostwald ripening rate of $0.12 \pm 0.06 \mathrm{~nm}^{3} / \mu \mathrm{sec}$, which favourably compares to the $0.17 \pm 0.01 \mathrm{~nm}^{3} / \mu \mathrm{sec}$ value obtained from the simulations of Figure 5. Thus, we conclude that our simulation approach and set up are suitable for reproducing Ostwald ripening for the benzene-water system, yielding results that are in satisfactory agreement with the LSW theoretical predictions. 


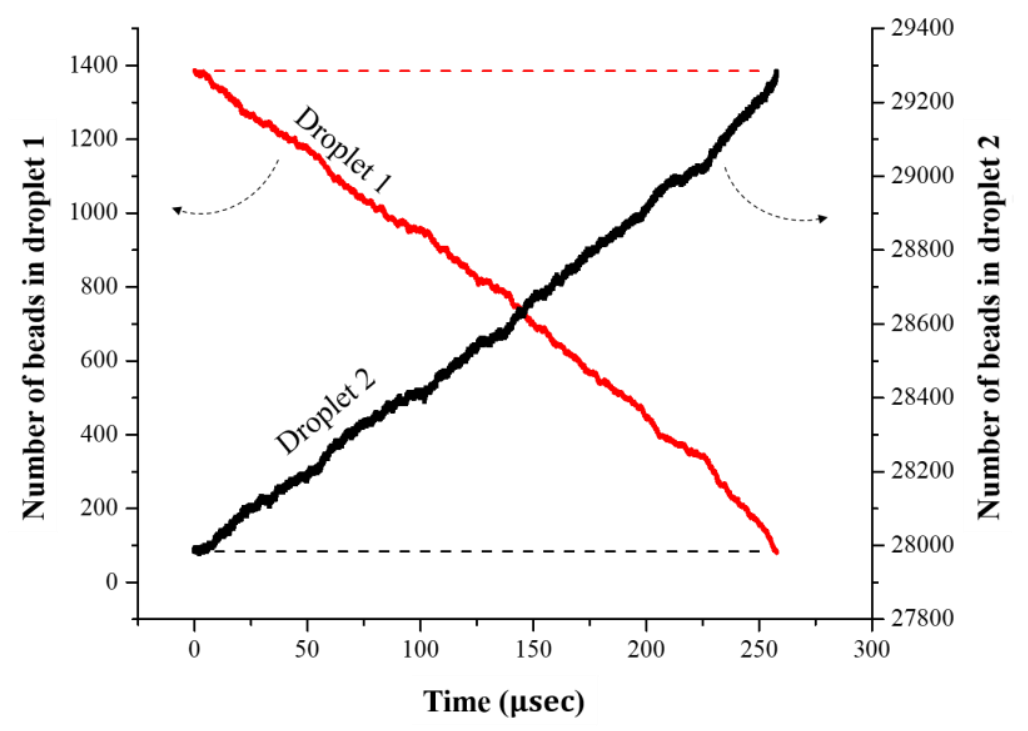

Figure 4. Number of beads belonging to the two droplets during Ostwald ripening simulations. The left and right axis show the number of beads in droplet 1 (small) and droplet 2 (large), respectively. The horizontal dashed lines indicate the droplets size at the beginning of the simulations.

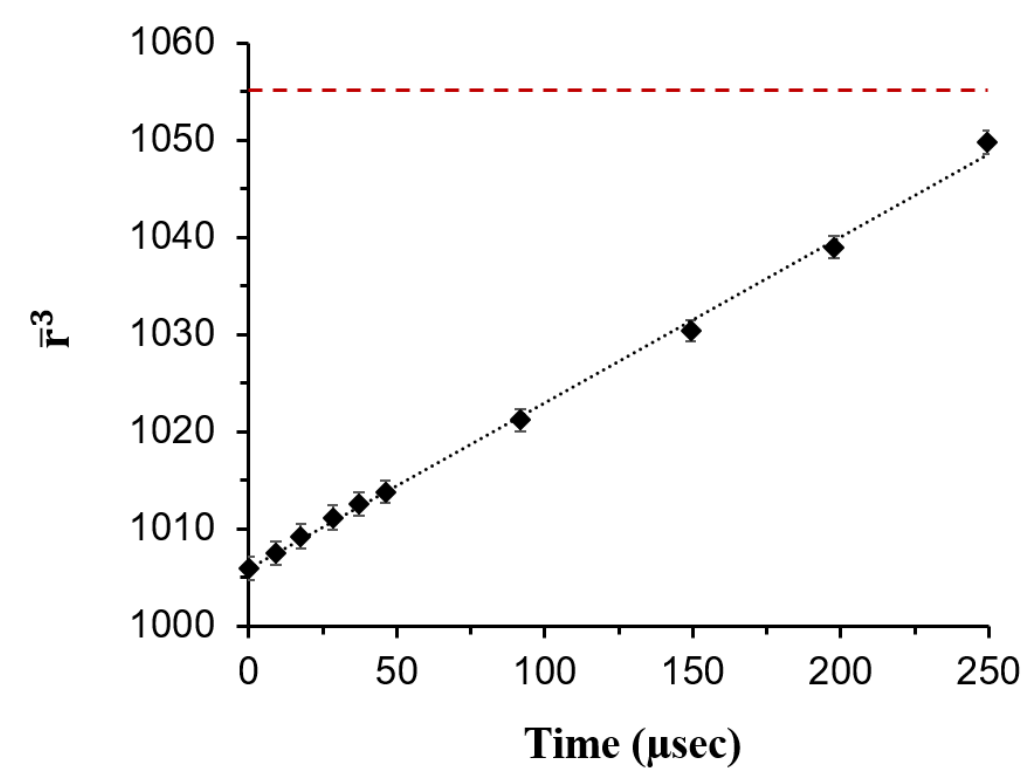

Figure 5. Cube of the mean radius of droplet 2 as a function of time. The horizontal dashed line indicates the final radius of the droplet, when the first droplet has disappeared. 
Table 2. Ostwald ripening rate in $\mathrm{nm}^{3} / \mu \mathrm{sec}$ of benzene-in-water emulsion estimated from simulation (this work), experiment and theory. ${ }^{24}$

$\begin{array}{cc}\text { Results from } & \text { Ostwald ripening rate }(\omega) \\ \text { Simulations (Figure 5) } & 0.17 \pm 0.01 \\ \text { Experiment (Literature) } & 3.1 \\ \mathrm{LSW} \text { theory at } 20^{\circ} \mathrm{C} \text { (Literature) } & 2.2 \\ \mathrm{LSW} \text { theory at } 25^{\circ} \mathrm{C} \text { (Estimated here) } & 1.72 \\ \mathrm{LSW} \text { theory using parameters from simulations } & 0.12 \pm 0.06\end{array}$

Table 3. Benzene solubility and diffusivity in water at $25^{\circ} \mathrm{C}$ (experiments ${ }^{61}$ and simulations).

$\begin{array}{ccc} & \text { Experimental at } 25^{\circ} \mathrm{C} . & \text { Simulation } \\ & 2.28 \times 10^{-2} \mathrm{M} & 7.4 \pm 4 \times 10^{-5} \mathrm{~m}^{3} / \mathrm{m}^{3} \\ \text { Solubility } & \equiv 2 \times 10^{-3} \mathrm{~m}^{3} / \mathrm{m}^{3} & \\ \text { Diffusivity } & 8.1 \times 10^{-6} \mathrm{~cm}^{2} / \mathrm{s} & 1.7 \pm 0.02 \times 10^{-5} \mathrm{~cm}^{2} / \mathrm{s}\end{array}$

\subsection{Characteristics of the surfactants' interfacial films}

The rest of this study will focus on the effect of two surfactants on Ostwald ripening. We started by estimating the number of surfactant molecules needed to fully cover each of the two droplets. A large number of surfactants (2500 H1T2 and 1600 H1T2) were added to bulk water. Starting with the initial configurations showed in Figure 3, the surfactants first form micelles. These micelles tend to gather oil beads from both oil droplets. This is evident for H1T2 surfactants in the results discussed later (section 3.4). The micelles are then driven to the oil droplet/water interface. These observations appear consistent with results from Karaborni and co-workers, ${ }^{10,11}$ who observed three mechanisms of surfactant adsorption on droplets. The first mechanism involves the adsorption of monomeric surfactant molecules, the second is the coalescence between the micelle and oil droplets, and the third is the exchange of surfactant molecules through micelle/oil collisions.

As the simulations progressed, we monitored the amounts of surfactants adsorbed on the two droplets. Representative results are shown in Figure 6. From the plateaus, it is estimated 
that $\sim 313$ H1T2 surfactants are needed to saturate droplet 1 and $\sim 1993$ to saturate droplet 2. The correspondent values for H3T2 surfactants are 194 and 1114.

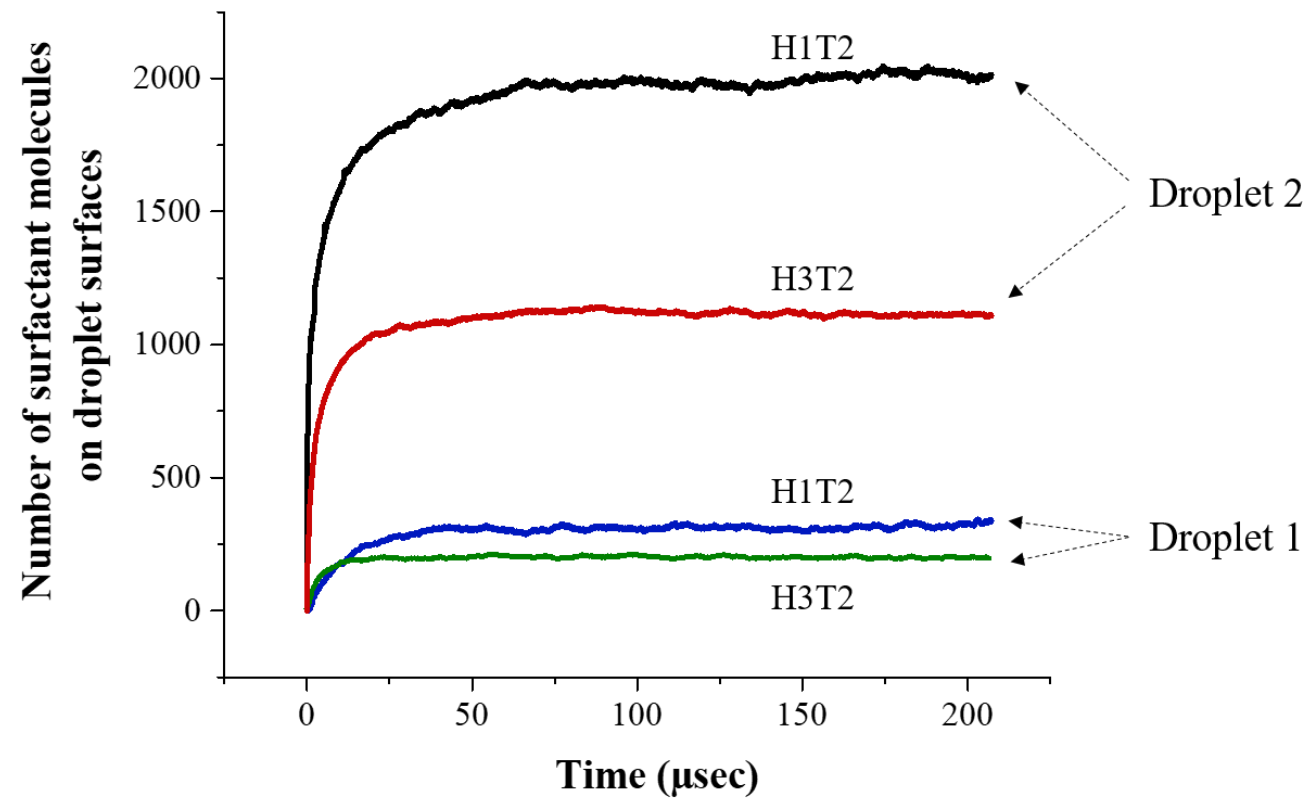

Figure 6. Number of surfactants molecules adsorbed to each droplet when 2500 and 1600 molecules of H1T2 and H3T2 surfactants are added, respectively.

From the results shown in Figure 6, it was determined that, in total, $~ 2300$ H1T2 and 1310 H3T2 surfactants saturate both droplet surfaces. In agreement with experimental observations, ${ }^{16}$ the surfactants with shorter hydrophilic groups can more densely pack at the droplet surfaces compared to the surfactant with longer head-groups. We observe a slight deformation in the droplet in the presence of H1T2 surfactants (see Figure 7, where the cross sectional views are shown together with the covered droplets). 
a)

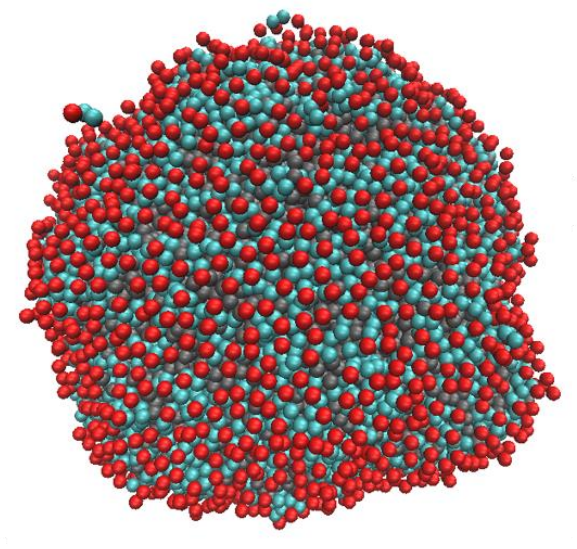

c)

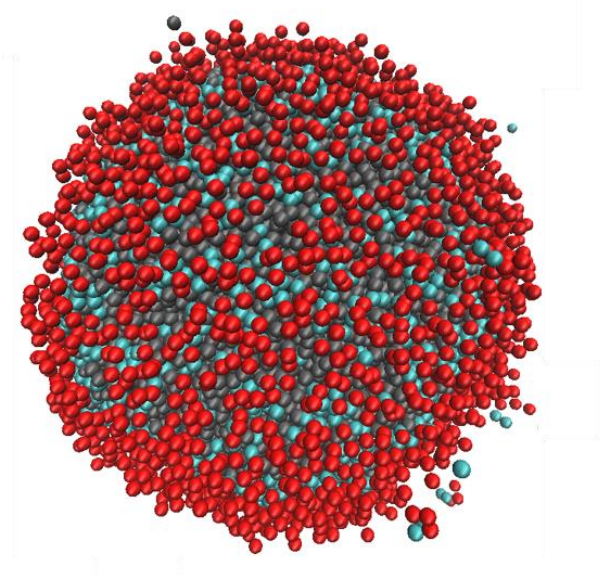

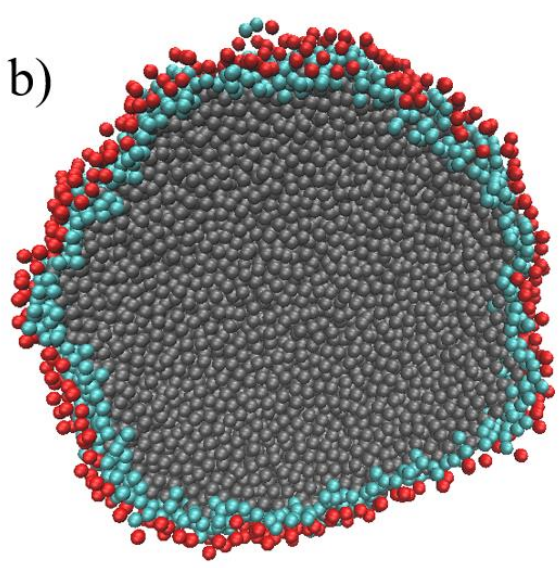

d)

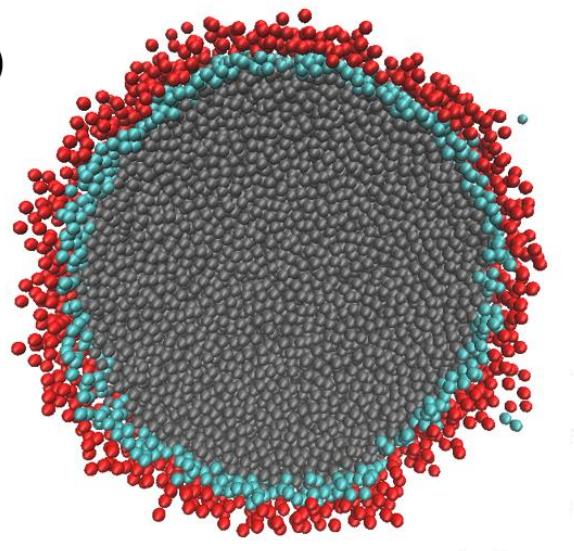

Figure 7. Droplet 2 saturated with $~ 1993$ H1T2 (top) and 1114 H3T2 surfactants (bottom). Panels (b) and (d) provide cross-sectional views of the droplets.

\subsection{Surfactants Effects on Ostwald Ripening}

We simulated Ostwald ripening in the presence of 2000 and 1300 molecules of H1T2 and H3T2 surfactants, respectively. These concentrations yield interfacial films just below saturation. These conditions were chosen to limit the likelihood of micelles formation, once the surfactants are adsorbed on the droplets. In Figure 8, we show the two systems in the presence of H1T2 and H3T2 surfactants, once the surfactants have been adsorbed on both droplets. Visual inspection confirms that micelles are not present. 
a)

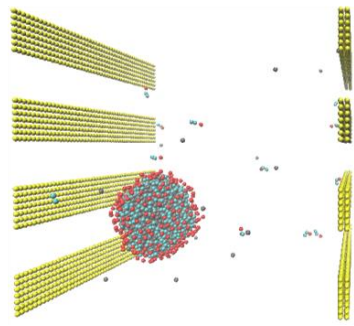

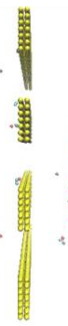

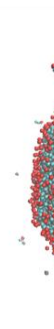

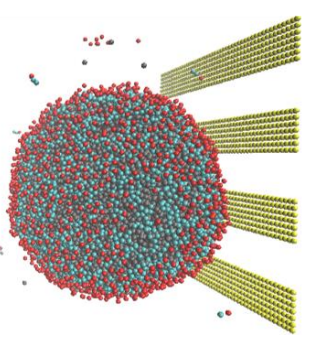

b)

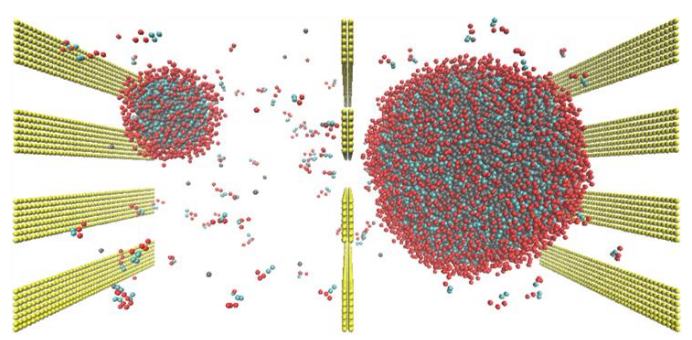

Figure 8. Simulated systems with a) 2000 H1T2 and b) 1300 H3T2 surfactants adsorbed on the benzene droplets.

In Figure 9, we report the number of oil beads in the two droplets as a function of simulation time. It should be noted that, because the surfactants delayed Ostwald ripening, it was not possible to continue our simulations until droplet 1 completely vanished. To be consistent with the results presented in Section 3.1, we conducted the simulations in presence of surfactants for up to $262 \mu \mathrm{sec}$. The results are compared to those with no surfactants (Figure 4). Visual observation confirms that both surfactants slow down Ostwald ripening, as evidenced by the fact that the curves change slope after the complete adsorption of surfactants on the droplets. To quantify the change in Ostwald ripening rate, the increase in the cube of the mean radius of droplet 2 is plotted as a function of time (Figure 10), and the ripening rate is extracted from fitting the data after complete adsorption of surfactants on the droplet surfaces. Table 4 presents the average Ostwald ripening rate as obtained from the different simulations.

The simulation results show that the surfactants reduce the Ostwald ripening rate from $0.17 \pm 0.01 \quad \mathrm{~nm}^{3} / \mu \mathrm{sec} \quad$ to $\quad 0.02 \pm 0.01 \quad$ (H1T2) and $0.03 \pm 0.01 \mathrm{~nm}^{3} / \mu \mathrm{sec}(\mathrm{H} 3 \mathrm{~T} 2)$. These results yield a decrease in Ostwald ripening rate by a factor of $\sim 8$ and $\sim 5$ in presence of H1T2 and H3T2 surfactants, respectively. These results qualitatively agree with Kabalnov et al., ${ }^{24}$ whose experiments showed a decrease by a factor of 8 in Ostwald ripening rate when surfactants were added to their emulsions. This group reported that the dominant effect in reducing Ostwald ripening rate was due to the reduction in interfacial tension. Although our results generally agree with this conclusion, within the accuracy of our simulations, other effects might arise in the systems simulated. 

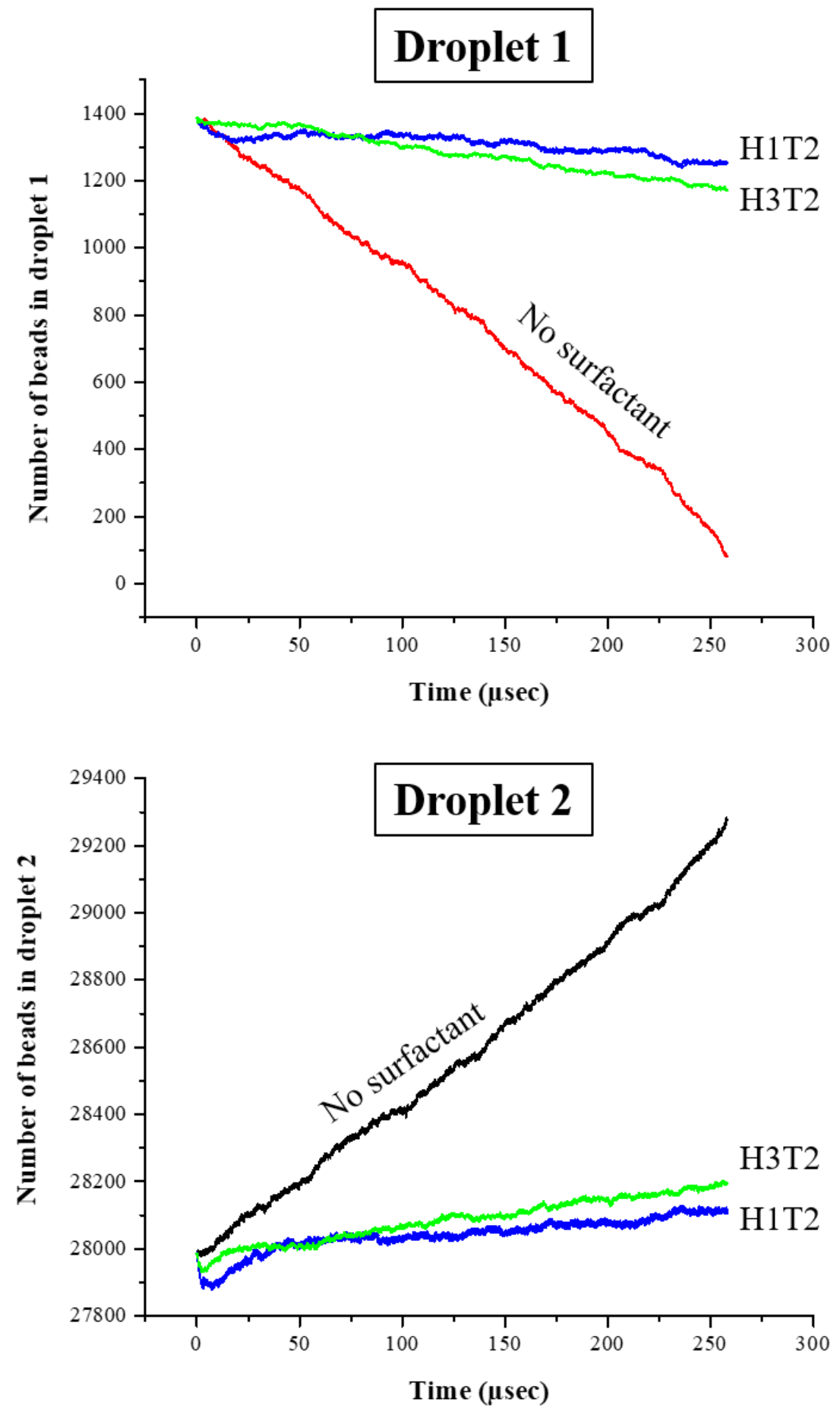

Figure 9. Change in the number of oil beads in the two droplets when either 2000 H1T2 or 1300 H3T2 surfactants are present, starting from the second initial configuration out of the three shown in Figure 3. The results are compared to those obtained when no surfactants are present in the simulation. 
Specifically, our prior simulations showed that the water-benzene interfacial tension at a planar interface is reduced from $30 \pm 8 \mathrm{mN} / \mathrm{m}$ to $17.4 \pm 8.5$ and $16.3 \pm 10 \mathrm{mN} / \mathrm{m}$ by H1T2 and H3T2 surfactants at high surface density, respectively. ${ }^{46}$ Using these interfacial tensions in Eq. (2) yields predicted Ostwald ripening rates of $0.07 \pm 0.04 \mathrm{~nm}^{3} / \mu \mathrm{sec}$ and $0.06 \pm 0.04$ $\mathrm{nm}^{3} / \mu$ sec for H1T2 and H3T2 surfactants, respectively, which are faster by a factor of $\sim 3$ and $\sim 2$, respectively, compared to our direct simulation results. Thus, in addition to reducing the interfacial tension, the surfactants must reduce Ostwald ripening rate via additional mechanisms. To further probe these systems, we conducted additional simulations in which the droplets were covered by fewer surfactants. The obtained Ostwald ripening rates, presented in the Supplementary Information (Table S1 and Figure S10), are in excellent agreement with the LSW predictions when the interfacial tension values at the correspondent surface coverage are used. We conclude that the reduction in interfacial tension is the primary mechanism by which surfactants reduce the Ostwald ripening rate, but only until a dense interfacial layer is obtained.

Experimental observations, summarized in the introduction, suggest that various mechanisms could determine how surfactants affect Ostwald ripening. For example, Han et al. ${ }^{16}$ and McClements at al. ${ }^{35}$ suggested that density and thickness of the surfactants film on the droplets surface might affect Ostwald ripening. These groups attempted to quantify the effect of the interfacial films formed by surfactants with different head-group length on Ostwald ripening. It is possible that once the surfactants simulated in our systems yield a dense surface film, barriers to Ostwald ripening in addition to interfacial tension reduction arise. For example, because our simulations show a slower Ostwald ripening rate in the presence of H1T2 surfactants, which yield higher surface density on the two droplets compared to H3T2, it is possible that our results reflect the possibility that H1T2 surfactants introduce a stronger resistance to benzene beads from diffusing across the interfacial film at the droplet-water interface, which seems to be consistent with the experiments just summarised. To provide a quantitative comparison against the results reported by Han et al., ${ }^{16}$ models are needed to replicate the molecular-level features of the surfactant-laden interfaces, especially when surfactants with different headgroups are considered.

For completeness, it should be pointed out that, as our simulations progress, the surfactant films on the droplets change because the surface density increases as a droplet shrinks and decreases as a droplet grows. In Figure S9 in SI we show how the number of surfactants adsorbed on the two droplets change as a function of simulation time. This affects surface packing, which directly affects the ability of oil beads to transfer to the continuous aqueous 
phase, as just discussed. However, within the conditions considered here, these changes do not affect significantly the results presented.
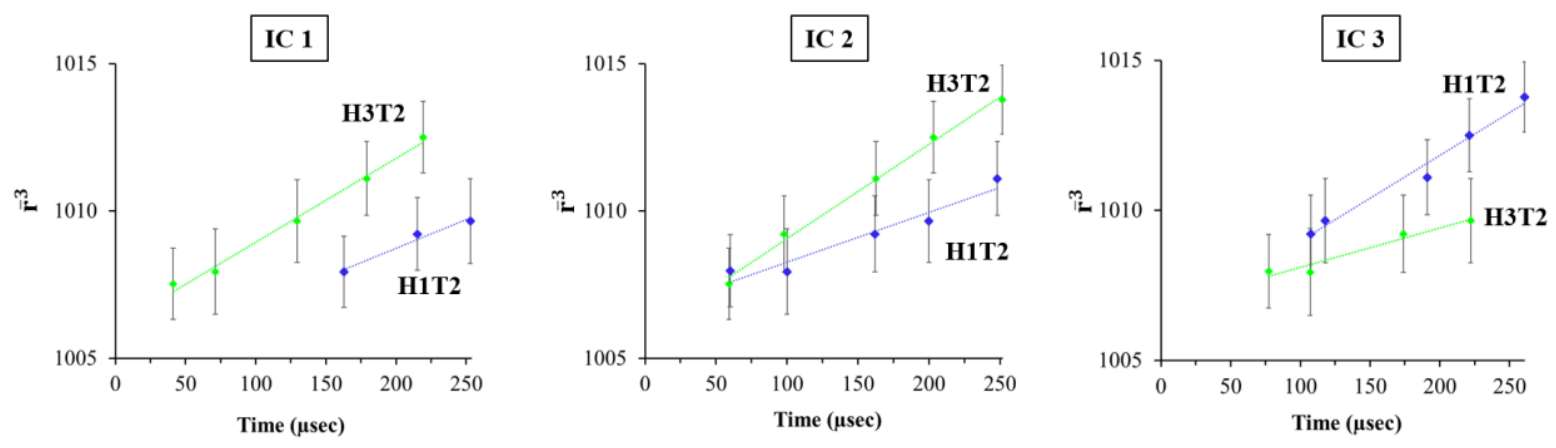

Figure 10. Cube of the mean radius of droplet 2 as a function of time in the presence of 2000 H1T2 and $1300 \mathrm{H} 3 \mathrm{~T} 2$ surfactants, starting from the three initial configurations (IC) shown in Figure 3.

Table 4. Ostwald ripening rate in $\mathrm{nm}^{3} / \mu \mathrm{sec}$ estimated from simulations and theory in the presence of H1T2 and H3T2 surfactants.

Results from

H1T2

H3T2

Simulations (Figure 10)

LSW using the reduced interfacial tension
$0.03 \pm 0.01$

$0.07 \pm 0.04$

$0.06 \pm 0.04$

\subsection{Ostwald ripening in the presence of micelles}

It has been suggested that the presence of micelles could affect Ostwald ripening. ${ }^{29,}{ }^{34}$ In one of our simulations, conducted in the presence of H1T2 surfactants, one swollen micelle formed because of the coalescence between small micelles and the accumulation of oil beads (see Figure 11). The swollen micelle was not able to traverse the membrane and coalesce with droplet 2. While the micelle was present, the sum of the number of benzene beads plus surfactants on droplet 1 is constant (Figure 12), while droplet 2 seems to grow at the expense of the micelle. In fact, detailed analysis (see Figure S11 in SI) shows a more complex molecular 
exchange; the benzene beads transferred because of Ostwald ripening between the micelle and droplet 1 , while some surfactant molecules diffused from droplet 1 to droplet 2 . Some benzene beads also diffused from the micelle to droplet 2. Once the micelle disappeared (vertical line in Figure 12), Ostwald ripening started between droplets 1 and 2, as discussed in Sec. 3.3. Although this detailed molecular exchange occurring in presence of a micelle is difficult to observe experimentally, the overall change in the size of droplet 2 in the presence of the micelle seems to be in qualitative agreement with the experimental observations by Ariyaprakai and Dungan, ${ }^{34}$ who however reported a much more pronounced effect due to micelles. Depending on surfactant type and concentration, the Ostwald ripening rate increased by a factor up to 50 . The difference between simulated and experimental results is probably due to the high surfactant concentration used in the experiments. In fact, as the surfactant concentration increases, the experimental Ostwald ripening rate increases as well. It is possible that when many micelles are present, they enhance Ostwald ripening rate by increasing the oil solubility in water (due to the small micellar size). It is also possible that micelles contribute to enhance the transport of oil from small to large droplets, via their diffusion. This was not observed in our simulations, by design, mostly because of the low surfactant concentration used.

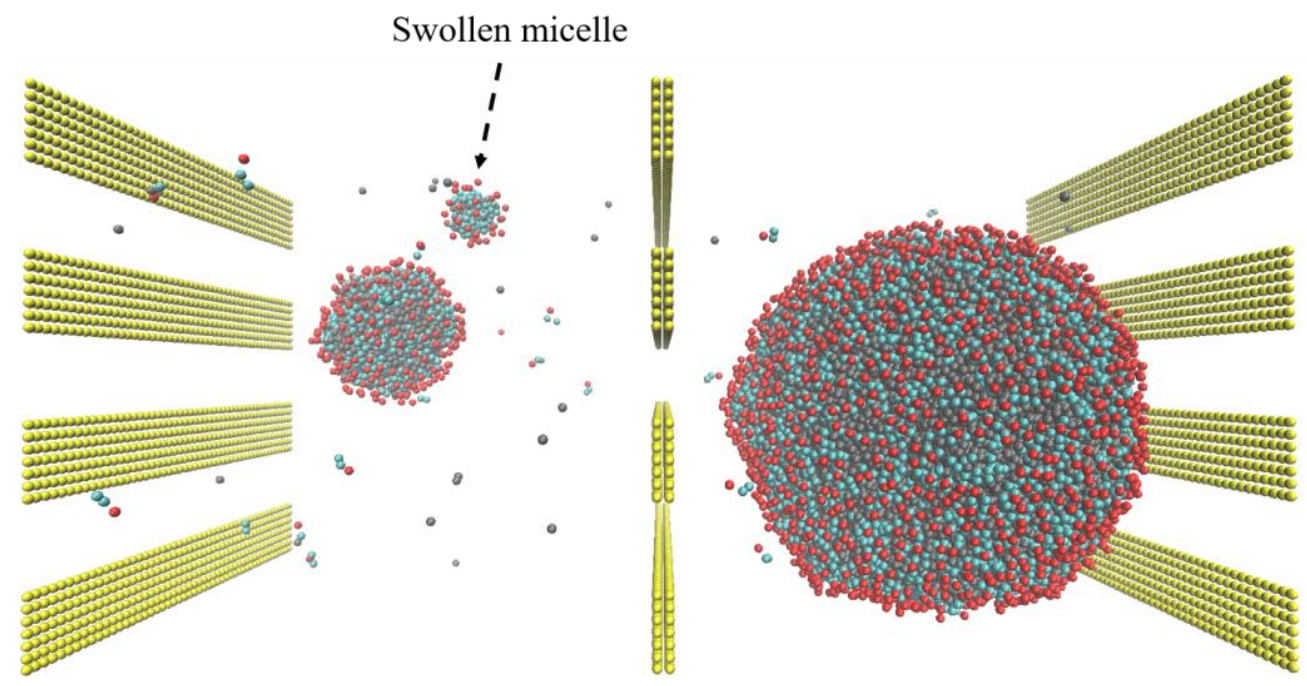

Figure 11. Simulation snapshots showing droplets 1 and 2, both covered by the H1T2 surfactant, as well as a swollen micelle, highlighted by an arrow. 


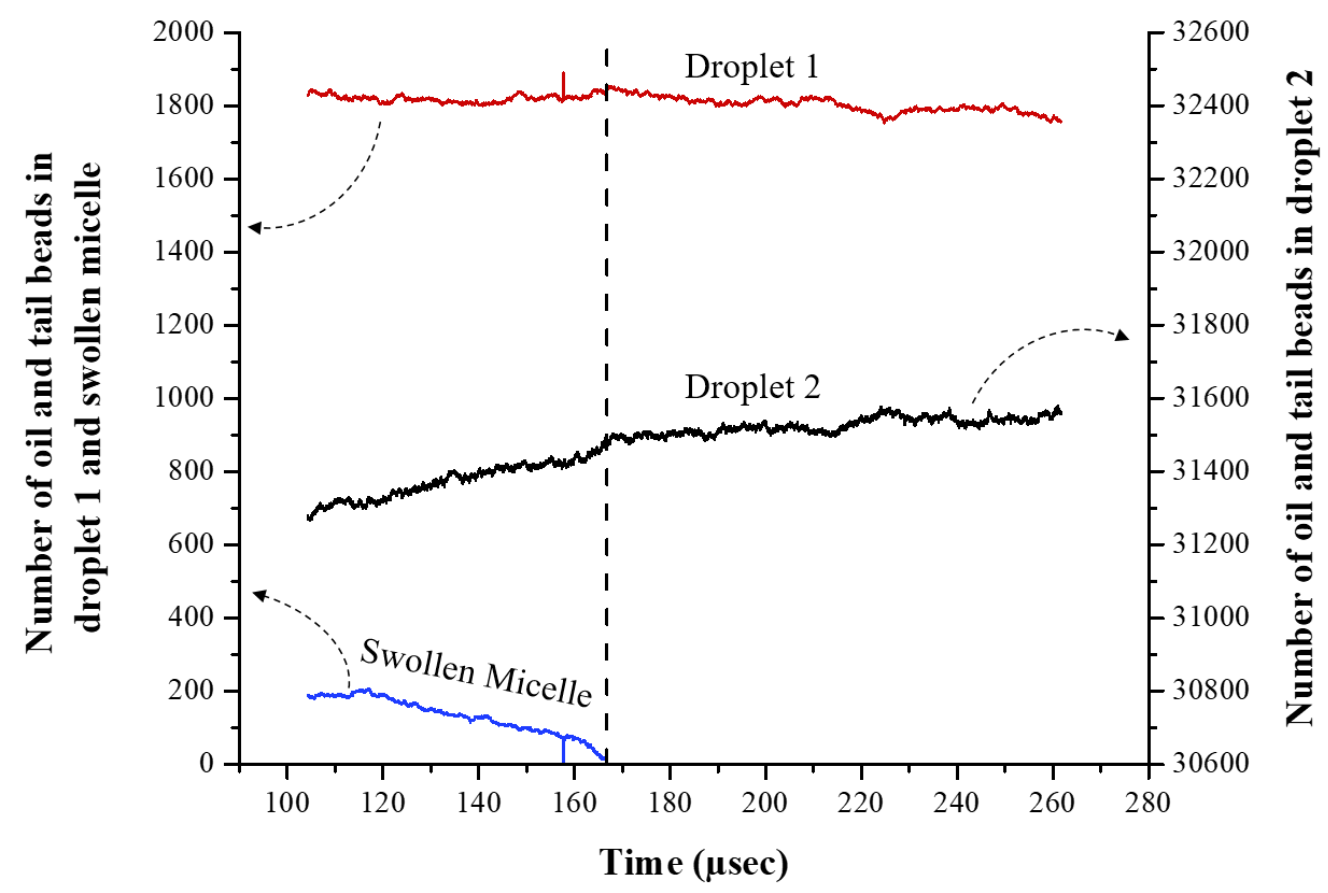

Figure 12. Number of oil + number of tail beads in droplet 1 (red), droplet 2 (black) and in the swollen micelle (blue) as a function of simulation time. Note that, while the number of beads in droplet 2 increase, those in droplet 1 remain approximately constant until the micelle completely disappear. This condition is highlighted by the dashed vertical black line.

\section{Conclusions}

Ostwald ripening is a natural mechanism responsible for the destabilisation of emulsions. Its effects are important, sometimes deleterious, for a variety of industrial applications, ranging from the formulation of specialty chemicals to foodstuff, as well as pharmaceuticals. Molecular models able to quantitatively relate the molecular features of surfactants and other chemical compounds used in a formulation to the Ostwald ripening rate could facilitate the prediction of the long-term stability of industrial formulations. Because many mechanisms contribute to Ostwald ripening, as a first step towards a general approach to quantify Ostwald ripening rates in surfactants-stabilised emulsions, we describe here a simulation approach, based on the coarse-grained Dissipative Particle Dynamics formalism, which is able to qualitatively replicate Ostwald ripening rates for benzene droplets dispersed in water. Nearly quantitative reproduction of experimental observations is achieved when simulation results for solubility and diffusivity of benzene in water are used. 
When applied to systems containing surfactants, the approach shows that the Ostwald ripening rate is reduced in large part because the surfactants reduce the benzene-water interfacial tension. However, other phenomena might also be important, especially when the surfactants yield a compact interfacial layer on the droplets. These additional phenomena include the resistance to transfer of benzene from the oil droplet to the continuous aqueous phase, due to the surfactant film at the benzene-water interface, as well as the possible formation of micelles. The former mechanism delays Ostwald ripening rate, while the latter seems to speed it up.

The results presented prove that coarse-grained simulations are able to capture the essential phenomena leading to Ostwald ripening. For future practical applications of the methods described here, it should be emphasized that the simulations allow us to identify the molecular mechanisms responsible for either speeding up or delaying Ostwald ripening. A detailed analysis of the effect of micelles on Ostwald ripening is feasible using the set up presented here, but it requires simulating systems at high surfactant concentration. When implementing coarse-grained simulations to study kinetic phenomena, one should remember that soft potentials, such as those employed in dissipative particle dynamics, tend to over-estimate diffusion coefficients, which needs to be accounted for when interpreting the simulation results to match experimental observations.

\section{Supporting Information}

The molecular exchange due to Ostwald ripening starting from different initial configurations; the molecular exchange when varying the curvature of droplet 2; the number of both oil beards within and H1T2 and H3T2 surfactants on the surface of the two droplets as a function of simulation time starting ; the interfacial tension at different surface densities and the resultant Ostwald ripening rate from simulations and theory. These results are available in Supporting Information free of charge from the ACS Publications website at DOI:

\section{Acknowledgments}

Generous allocations of computing time were provided by the University College London Research Computing Platforms Support (LEGION). The authors acknowledge financial support from the Department of Chemical Engineering at the University College London. The Authors 
acknowledge stimulating discussions with Prof. John Shaw, whose sabbatical at the University College London was supported by The Leverhulme Trust, via grant agreement number VP2-2017023. The Authors are grateful to Ian McRobbie, of Innospec, for stimulating discussions and encouragement.

\section{References}

(1) Binks, B. P.; Services, R. S. o. C. I. Modern Aspects of Emulsion Science. Royal Society of Chemistry: 1998.

(2) Alvarez, F.; Flores, E. A.; Castro, L. V.; Hernandez, J. G.; Lopez, A.; Vazquez, F. Dissipative Particle Dynamics (Dpd) Study of Crude Oil-Water Emulsions in the Presence of a Functionalized Co-Polymer. Energy Fuels. 2011, 25, 562-567.

(3) Binks, B. P.; Lumsdon, S. O. Pickering Emulsions Stabilized by Monodisperse Latex Particles: Effects of Particle Size. Langmuir. 2001, 17, 4540-4547.

(4) Huibers, P. D. T.; Shah, D. O. Evidence for Synergism in Nonionic Surfactant Mixtures: Enhancement of Solubilization in Water-in-Oil Microemulsions. Langmuir. 1997, 13, 5762-5765. (5) Lin, S. L.; Xu, M. Y.; Yang, Z. R. Dissipative Particle Dynamics Study on the Mesostructures of NOctadecane/Water Emulsion with Alternating Styrene-Maleic Acid Copolymers as Emulsifier. Soft Matter. 2012, 8, 375-384.

(6) Lopetinsky, R., Masliyah, J., \& Xu, Z. , Solids-Stabilized Emulsions: A Review. In Colloidal Particles at Liquid Interfaces Horozov, B. P. B. T. S., Ed. Cambridge University Press: Cambridge, 2006; pp 186224.

(7) Binks, B. P. Particles as Surfactants - Similarities and Differences. Curr. Opin. Colloid Interf. Sci. 2002, 7, 21-41.

(8) Song, X. Y.; Shi, P.; Duan, M.; Fang, S. W.; Ma, Y. Z. Investigation of Demulsification Efficiency in Water-in-Crude Oil Emulsions Using Dissipative Particle Dynamics. RSC Adv. 2015, 5, 62971-62981.

(9) Rekvig, L.; Frenkel, D. Molecular Simulations of Droplet Coalescence in Oil/Water/Surfactant Systems. J. Chem. Phys. 2007, 127.

(10) Esselink, K.; Hilbers, P. A. J.; Vanos, N. M.; Smit, B.; Karaborni, S. Molecular-Dynamics Simulations of Model Oil/Water/Surfactant Systems. Colloid Surface A. 1994, 91, 155-167.

(11) Karaborni, S.; Vanos, N. M.; Esselink, K.; Hilbers, P. A. J. Molecular-Dynamics Simulations of Oil Solubilization in Surfactant Solutions. Langmuir. 1993, 9, 1175-1178.

(12) Mondal, S.; Ghosh, S.; De, S. Atomistic Level Molecular Dynamics Simulation on the Solubilization Mechanism of Aromatic Molecules in Anionic Micelles. Rsc Adv. 2015, 5, $104493-$ 104501.

(13) Li, S. Y.; Zhang, X. R.; Dong, W.; Wang, W. C. Computer Simulations of Solute Exchange Using Micelles by a Collision-Driven Fusion Process. Langmuir. 2008, 24, 9344-9353.

(14) Kraska, T. Direct Observation of Single Ostwald Ripening Processes by Molecular Dynamics Simulation. J Phys Chem B. 2008, 112, 12408-12413.

(15) Lindfors, L.; Skantze, P.; Skantze, U.; Rasmusson, M.; Zackrisson, A.; Olsson, U. Amorphous Drug Nanosuspensions. 1. Inhibition of Ostwald Ripening. Langmuir. 2006, 22, 906-910.

(16) Han, S. W.; Song, H. Y.; Moon, T. W.; Choi, S. J. Influence of Emulsion Interfacial Membrane Characteristics on Ostwald Ripening in a Model Emulsion. Food Chem. 2018, 242, 91-97.

(17) McClements, D. J.; Henson, L.; Popplewell, L. M.; Decker, E. A.; Choi, S. J. Inhibition of Ostwald Ripening in Model Beverage Emulsions by Addition of Poorly Water Soluble Triglyceride Oils. J. Food Sci. 2012, 77, C33-C38. 
(18) Lim, S. S.; Baik, M. Y.; Decker, E. A.; Henson, L.; Popplewell, L. M.; McClements, D. J.; Choi, S. J. Stabilization of Orange Oil-in-Water Emulsions: A New Role for Ester Gum as an Ostwald Ripening Inhibitor. Food Chem. 2011, 128, 1023-1028.

(19) Ziani, K.; Fang, Y.; McClements, D. J. Fabrication and Stability of Colloidal Delivery Systems for Flavor Oils: Effect of Composition and Storage Conditions. Food Res. Int. 2012, 46, 209-216.

(20) Rao, J.; McClements, D. J. Food-Grade Microemulsions and Nanoemulsions: Role of Oil Phase Composition on Formation and Stability. Food Hydrocolloid. 2012, 29, 326-334.

(21) Thomson, W. W. Thomson, Philos. Mag. 42, 448 (1871). Philos. Mag. 1871, 42, 448.

(22) Lifshitz, I. M.; Slyozov, V. V. The Kinetics of Precipitation from Supersaturated Solid Solutions. J. Phys. Chem. Solids. 1961, 19, 35-50.

(23) Wagner, C. Theorie Der Alterung Von Niederschlägen Durch Umlösen (Ostwald-Reifung). Z. Elektrochem. 1961, 65, 581-591.

(24) Kabalnov, A. S.; Pertzov, A. V.; Shchukin, E. D. Ostwald Ripening in Emulsions .1. Direct Observations of Ostwald Ripening in Emulsions. J. Colloid Interf. Sci. 1987, 118, 590-597.

(25) Voorhees, P. W. The Theory of Ostwald Ripening. J. Stat. Phys. 1985, 38, 231-252.

(26) Kabalnov, A.; Makarov, K.; Pertzov, A.; Shchukin, E. Ostwald Ripening in Emulsions: 2. Ostwald Ripening in Hydrocarbon Emulsions: Experimental Verification of Equation for Absolute Rates. J. Colloid Interf. Sci. 1990, 138, 98-104.

(27) Kabalnov, A. S. Can Micelles Mediate a Mass Transfer between Oil Droplets? Langmuir. 1994, 10, 680-684.

(28) Taylor, P.; Ottewill, R. H. The Formation and Aging Rates of Oil-in-Water Miniemulsions. Colloid Surface A. 1994, 88, 303-316.

(29) Weiss, J.; Coupland, J. N.; Brathwaite, D.; McClements, D. J. Influence of Molecular Structure of Hydrocarbon Emulsion Droplets on Their Solubilization in Nonionic Surfactant Micelles. Colloid Surface A. 1997, 121, 53-60.

(30) Weiss, J.; Canceliere, C.; McClements, D. J. Mass Transport Phenomena in Oil-in-Water Emulsions Containing Surfactant Micelles: Ostwald Ripening. Langmuir. 2000, 16, 6833-6838.

(31) Weiss, J.; McClements, D. J. Mass Transport Phenomena in Oil-in-Water Emulsions Containing Surfactant Micelles: Solubilization. Langmuir. 2000, 16, 5879-5883.

(32) Chanamai, R.; Horn, G.; McClements, D. J. Influence of Oil Polarity on Droplet Growth in Oil-inWater Emulsions Stabilized by a Weakly Adsorbing Biopolymer or a Nonionic Surfactant. J. Colloid Interf. Sci. 2002, 247, 167-176.

(33) Weiss, J.; Herrmann, N.; McClements, D. J. Ostwald Ripening of Hydrocarbon Emulsion Droplets in Surfactant Solutions. Langmuir. 1999, 15, 6652-6657.

(34) Ariyaprakai, S.; Dungan, S. R. Influence of Surfactant Structure on the Contribution of Micelles to Ostwald Ripening in Oil-in-Water Emulsions. J. Colloid Interf. Sci. 2010, 343, 102-108.

(35) Mun, S. H.; McClements, D. J. Influence of Interfacial Characteristics on Ostwald Ripening in Hydrocarbon Oil-in-Water Emulsions. Langmuir. 2006, 22, 1551-1554.

(36) Jung, J.; Lee, J.; Kim, J. S. Cluster Growth Mechanisms in Lennard-Jones Fluids: A Comparison between Molecular Dynamics and Brownian Dynamics Simulations. Chem Phys. 2015, 449, 1-9.

(37) Jung, J.; Jang, E.; Shoaib, M. A.; Jo, K.; Kim, J. S. Droplet Formation and Growth inside a Polymer Network: A Molecular Dynamics Simulation Study. J Chem Phys. 2016, 144.

(38) Zhang, H. Z.; Banfield, J. F. Aggregation, Coarsening, and Phase Transformation in Zns Nanoparticles Studied by Molecular Dynamics Simulations. Nano Lett. 2004, 4, 713-718.

(39) Fu, X. J.; Cueto-Felgueroso, L.; Juanes, R. Thermodynamic Coarsening Arrested by Viscous Fingering in Partially Miscible Binary Mixtures. Physical Review E. 2016, 94.

(40) Fan, D. N.; Chen, S. P.; Chen, L. Q.; Voorhees, P. W. Phase-Field Simulation of 2-D Ostwald Ripening in the High Volume Fraction Regime. Acta Mater. 2002, 50, 1895-1907.

(41) Fan, H.; Striolo, A. Mechanistic Study of Droplets Coalescence in Pickering Emulsions. Soft Matter. 2012, 8, 9533-9538. 
(42) Fan, H.; Striolo, A. Nanoparticle Effects on the Water-Oil Interfacial Tension. Phys. Rev. E. 2012, 86, 051610.

(43) Sicard, F.; Striolo, A. Numerical Analysis of Pickering Emulsion Stability: Insights from Abmd Simulations. FARADAY DISCUSS. 2016, 191, 287-304.

(44) Luu, X. C.; Striolo, A. Ellipsoidal Janus Nanoparticles Assembled at Spherical Oil/Water Interfaces. J. Phys. Chem. B. 2014, 118, 13737-13743.

(45) Luu, X.-C.; Yu, J.; Striolo, A. Nanoparticles Adsorbed at the Water/Oil Interface: Coverage and Composition Effects on Structure and Diffusion. Langmuir. 2013, 29, 7221-7228.

(46) Khedr, A.; Striolo, A. Dpd Parameters Estimation for Simultaneously Simulating Water-Oil Interfaces and Aqueous Nonionic Surfactants. J. Chem. Theory Comput. 2018, 14, 6460-6471.

(47) Suttipong, M.; Grady, B. P.; Striolo, A. Self-Assembled Surfactants on Patterned Surfaces:

Confinement and Cooperative Effects on Aggregate Morphology. Phys. Chem. Chem. Phys. 2014, 16, 16388-16398.

(48) Suttipong, M.; Grady, B. P.; Striolo, A. Surfactant Aggregates Templated by Lateral Confinement. J. Phys. Chem. B. 2015, 119, 5467-5474.

(49) Suttipong, M.; Grady, B. P.; Striolo, A. Surfactants Adsorption on Crossing Stripes and Steps. Soft Matter. 2017, 13, 862-874.

(50) Hoogerbrugge, P. J.; Koelman, J. M. V. A. Simulating Microscopic Hydrodynamic Phenomena with Dissipative Particle Dynamics. EUROPHYS. LETT. 1992, 19, 155.

(51) Frenkel, D.; Smit, B. Understanding Molecular Simulation 2nd ed.; Academic Press, Inc.: Orlando, Florida, 2002; Vol. 1, pp 465-476.

(52) Groot, R. D.; Warren, P. B. Dissipative Particle Dynamics: Bridging the Gap between Atomistic and Mesoscopic Simulation. J. Chem. Phys. 1997, 107, 4423-4435.

(53) Plimpton, S. Fast Parallel Algorithms for Short-Range Molecular-Dynamics. J. Comput. Phys. 1995, 117, 1-19.

(54) Groot, R. D.; Rabone, K. L. Mesoscopic Simulation of Cell Membrane Damage, Morphology Change and Rupture by Nonionic Surfactants. Biophys. J. 2001, 81, 725-736.

(55) Johnston, M. A.; Swope, W. C.; Jordan, K. E.; Warren, P. B.; Noro, M. G.; Bray, D. J.; Anderson, R. L. Toward a Standard Protocol for Micelle Simulation. J. Phys. Chem. B. 2016, 120, 6337-6351.

(56) Vymetal, J.; Vondrášek, J. i. Gyration-and Inertia-Tensor-Based Collective Coordinates for Metadynamics. Application on the Conformational Behavior of Polyalanine Peptides and Trp-Cage Folding. J. Phys. Chem. A. 2011, 115, 11455-11465.

(57) Sicard, F.; Striolo, A. Buckling in Armored Droplets. Nanoscale. 2017, 9, 8567-8572.

(58) Goicochea, A. G.; Romero-Bastida, M.; Lopez-Rendon, R. Dependence of Thermodynamic Properties of Model Systems on Some Dissipative Particle Dynamics Parameters. Mol. Phys. 2007, 105, 2375-2381.

(59) Tolman, R. C. The Effect of Droplet Size on Surface Tension. J Chem Phys. 1949, 17, 333-337.

(60) Sicard, F.; Toro-Mendoza, J.; Striolo, A. Nanoparticles Actively Fragment Armored Droplets. arXiv preprint arXiv:1905.02826. 2019.

(61) Todorov, P. D.; Kralchevsky, P. A.; Denkov, N. D.; Broze, G.; Mehreteab, A. Kinetics of Solubilization of N-Decane and Benzene by Micellar Solutions of Sodium Dodecyl Sulfate. J. Colloid Interf. Sci. 2002, 245, 371-382. 


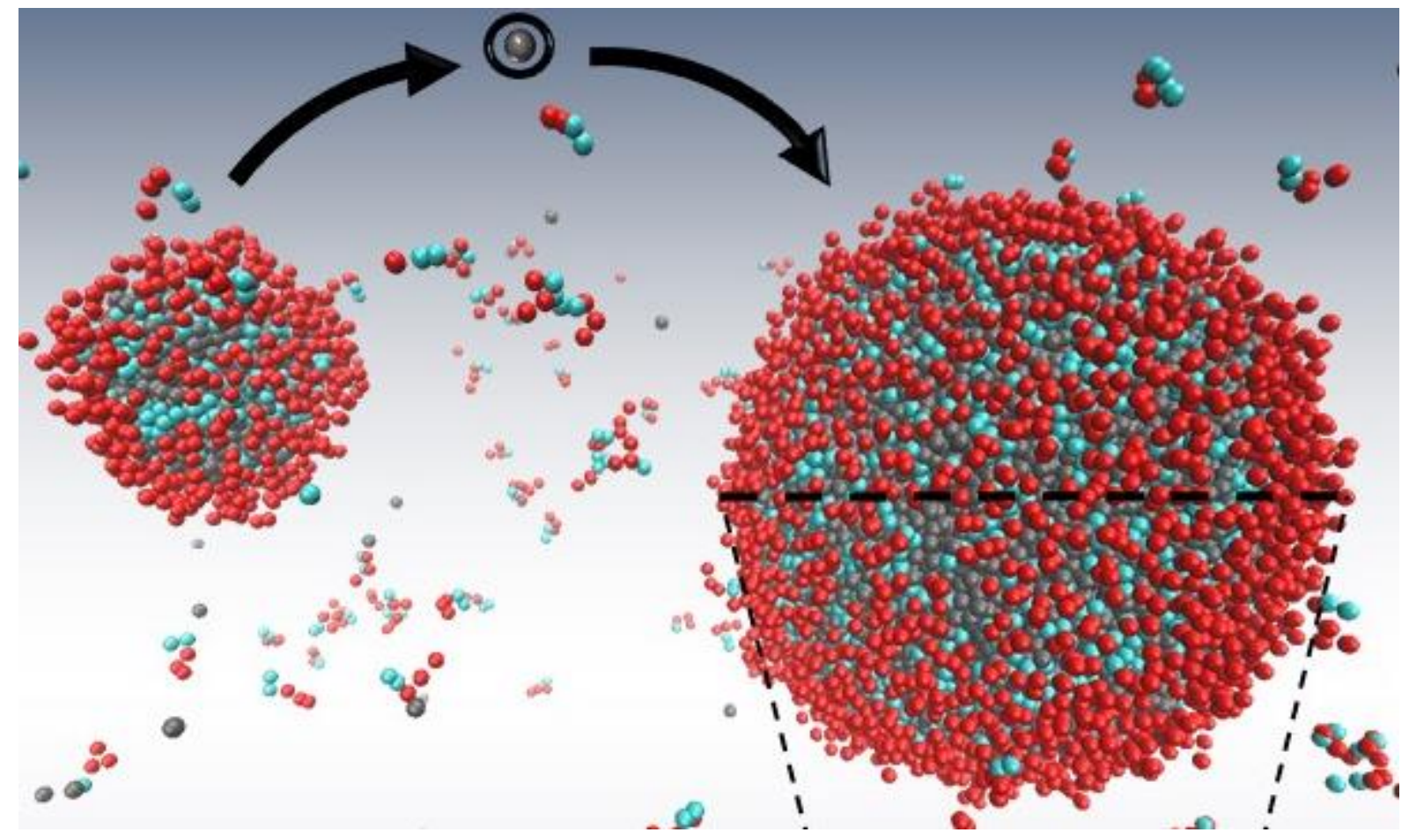

TOC graphic 